\title{
Mapping brucellosis risk in communities in the Republic of Armenia
}

\author{
Thibaud Porphyre ${ }^{1,2}$, Ronald Jackson ${ }^{2}$, Carola Sauter-Louis ${ }^{3}$, David Ward ${ }^{4}$, Grigor \\ Baghyan $^{5}$, Edik Stepanyan ${ }^{5}$ \\ ${ }^{1}$ CIRAD, UMR CIRAD-INRA 1309, Site de Duclos Prise d'Eau, Petit-Bourg, Guadeloupe, French West \\ Indies, France; ${ }^{2}$ EpiCentre, Institute of Veterinary, Animal and Biomedical Sciences, Massey University, \\ Palmerston North, New Zealand; ${ }^{3}$ Clinic for Ruminants, Ludwig-Maximilians-Munich University, Munich, \\ Germany; ${ }^{4}$ Food and Agriculture Organization of the United Nations, Rome, Italy; ${ }^{5}$ Food Safety and \\ Veterinary State Inspectorate, Ministry of Agriculture, Yerevan, Armenia
}

\begin{abstract}
We describe the geographical patterns and identified factors associated with serological evidence of brucellosis in ruminants in Armenian communities during 2006 and 2007. The data comprised the two first complete years of the current national test-and-slaughter control programme for cattle, sheep and goats. Overall, $29 \%$ and $21 \%$ of the 858 communities involved in this study reported brucellosis in their respective cattle and small ruminant populations. The national brucellosis control data showed a widespread and uneven distribution of brucellosis throughout the Republic of Armenia for both cattle and small ruminants. The geographical areas of greater risk of communities having seropositive animals were different for cattle and small ruminant populations but most of the associated factors were similar. Several areas where the likelihood of disease occurrence was predicted poorly by the statistical models were also identified. These latter findings are indicative of either less than perfect testing and reporting procedures or unexplained epidemiological factors operating in those particular areas. The analyses provided valuable insights into understanding the brucellosis epidemiology at the community level which operates in small ruminant and cattle populations, and identified priority areas for implementing targeted risk-based surveillance and disease control interventions.
\end{abstract}

Keywords: brucellosis, ruminants, epidemiology, risk mapping, Republic of Armenia.

\section{Introduction}

Brucellosis is a common zoonotic disease, infecting more than 500,000 humans each year worldwide, mainly in Central Asia and Middle East (Pappas et al., 2006). The infection constitutes an important public health and economic problem for developing countries as many households depend on

\footnotetext{
Corresponding author:

Ronald Jackson

EpiCentre

Institute of Veterinary Animal and Biomedical Sciences

Massey University

Palmerston North 4442, New Zealand

Tel. +646350 4529; Fax +64 63505716

E-mail: R.Jackson@massey.ac.nz
}

livestock products for their subsistence. It has a dual effect upon food security and poverty. Wastage in infected animals, through abortions and decreased milk production, results in fewer growing and productive animals, less food and fibre off-take from infected flocks and reduced income generation. In humans it manifests as a chronic debilitating disease, often with a prolonged convalescence phase, and requires lengthy courses of antibiotic treatment.

The Republic of Armenia is a relatively small country of $29,743 \mathrm{~km}^{2}$ with a population of approximately 3 million people. There are 520,018 urban and 258,648 rural households distributed among 863 community, town and city level administrative units recognised for veterinary administrative purposes. The average incidence of reported 
cases of brucellosis in humans from 1996 to 2005 was 3.57 per 100,000 and appeared to rise in 2006 and 2007 to 5.79 and 8.24 , respectively, per 100,000 (Anonymous, 2008). The distribution of human cases was approximately $84 \%$ in rural and $16 \%$ in urban communities. However, it is generally acknowledged that the true brucellosis incidence is much higher due to under-reporting caused by poor access to health care (Hovhannisyan, 2004; von Schoen-Angerer, 2004).

A national control programme based on test-andslaughter was instituted by the Food Safety \& State Veterinary Inspectorate (FS\&SVI) in the second half of 2005, but it encountered a variety of institutional and technical capacity constraints that limited its efficacy. The constraints included no permanent individual animal identification, no compensation, limited funding and possible between-laboratory variability in application and interpretation of the Rose Bengal plate agglutination test (RBT) among the Republic's 11 regional laboratories. The instituted programme aimed to test all cattle of breeding age twice yearly, and all breeding age male and female sheep and goats annually and to slaughter all animals that were confirmed positive at the Central Veterinary Laboratory. The set of test data generated during the control programme was seen as a useful source for analyses aimed at obtaining insights into some epidemiological aspects of the disease, in particular its spatial distribution and the relative importance of socio-economic and agricultural factors operating at community level which could conceivably be associated with evidence of livestock brucellosis.

This study used data from the Armenian test-andslaughter programme for an epidemiological investigation of serological evidence of brucellosis in ruminants in Armenian communities during all of 2006 and 2007. The objectives were: (i) to describe the community-level geographical patterns of brucellosis infection for large and small ruminant populations; (ii) to investigate some topographic, agricultural and socio-economic factors associated with brucellosis in Armenian communities; (iii) to iden- tify areas of greater probability of brucellosis infection; and (iv) to use that information to identify potential areas where efficacy of disease control interventions could be improved or where further epidemiological investigation are warranted.

\section{Material and methods}

Most of the livestock in Armenia is kept in small holdings for subsistence purposes but there are a small number of large enterprises which supply large towns and cities with meat and milk products. The average herd size was estimated to be 3.8 (range 0-250), 3.8 (range 0-308) and 0.25 (range 0-30) for cattle, sheep and goats, respectively (Jackson and Porphyre, 2008). Three per cent $(\mathrm{n}=133)$ of the holdings kept all three species and mixtures of cattle and sheep, cattle and goats and sheep and goats were kept in $17 \%(\mathrm{n}=755), 3.6 \%(\mathrm{n}=162)$ and $4.3 \%$ ( $\mathrm{n}=192)$, respectively. The number of cows per village averaged about 290 and ranged from 1 to 2,329 , while for small ruminants (i.e. sheep plus goats) the average number was 600 (range 0-9,838). Communal grazing around communities is a common practice and many flocks migrate from communities to high altitude pastures in the summer.

\section{Study design}

We used the serological test data attributed to the 863 communities that were enrolled in the first two complete years (2006 and 2007) of the national brucellosis test-and-slaughter control programme for cattle, sheep and goats. Communities were considered to have evidence of brucellosis if both a positive RBT test and a confirmatory complement fixation test (CFT) were returned for one or more animals in the community. Because the FS\&SVI practice at that time was to only record communities with seropositive animals in the central database, communities for which there were no records of disease in the central database were considered to have no evidence of the disease. The outcome of interest was the presence or absence of serological evidence 
of brucellosis in Armenian communities. Brucellosis status data based on that criterion, geographical coordinates, animal population and socio-economic details were thus available for 858 communities. Cattle were kept in all communities but fewer ( $\mathrm{n}=$ $816)$ reported keeping more than one sheep or goat.

\section{Predictor variables}

Putative socio-economic and agricultural descriptors were selected from United Nations Development Program (UNDP) and the Food and Agricultural Organization (FAO) of the United Nations records. We selected 35 descriptors from these databases (Table 1), involving six geographical (e.g. distance to market or to district capital), 17 socio-economic (e.g. number of households and inhabitants, proportions of ethnic groups) and 12 agricultural variables (e.g. total animal population, annual milk yield). These variables were either $\log _{10^{-}}$ transformed for normalisation or categorised to quartiles (agricultural land surface, average and maximum household agricultural land surface) or fixed values, e.g. proportion of ethnic Yezidi $(<10 \%, 11-50 \%$, and $>50 \%)$, proportion of ethnic Armenian $(<40 \%, 41-80 \%$, and $>80 \%)$, and presence/absence of ethnic Russians in communities.

We also hypothesised that disease occurrence could be affected by elevation ( $\mathrm{m}$ above sea level) and density of communities. Elevation was included because it was considered a possible surrogate indicator for husbandry practices at high altitudes where snow and low temperatures necessitate long periods of close confinement of animals in sheds, which consequentially impact on opportunities for disease transmission. Topographical data providing elevation data at $90 \mathrm{~m}$ resolution (International Centre for Tropical Agriculture, 2004) were extracted from the NASA Shuttle Radar Topographic Mission (SRTM) digital elevation data, version 3. We included density of communities based on an assumption that opportunities for transmission of disease among closely clustered communities might be influenced by shared grazing and ease of move-
Table 1. List of variables derived from UNDP and FAO reports which were tested in mixed-effects logistic regression models to identify factors associated with the odds of communities having at least one brucellosis-seropositive cattle or small ruminant.

Variables

Community distance from capital city $(\mathrm{km})$

Community distance from (district of) Marz center $(\mathrm{km})$

Community distance from former district center $(\mathrm{km})$

Community distance from nearby large trading market $(\mathrm{km})$

Community distance from nearby large farmers market $(\mathrm{km})$

Community location to Armenian border: (1) not borderline,

(2) near border with Iran, (3) near border with Georgia,

(4) near border with Turkey, (5) near border with Azerbaijan

Proportion of non-poor inhabitants in the community (\%)

Proportion of poor inhabitants in the community (\%)

Proportion of destitute in the community $(\%)$

Number of settlements in the community

Total permanent population, persons

Total present population, persons

Total present females, persons

Number of total permanent households

Number of total present households

Number of total present households residing in temporary dwellings (wagons, "domiks")

Proportion of Armenian in the community (\%)

Proportion of Russian in the community (\%)

Proportion of Yezidi in the community (\%)

Proportion of Kurd in the community (\%)

Proportion of Assyrian in the community (\%)

Proportion of Greek in the community (\%)

Proportion of other nationalities in the community (\%)

Number of agricultural self-employment

Number of persons who have paid work in agriculture

Number of persons who are occupied in agriculture

Administrative territory of the community (ha)

Total community land surface (ha)

Agricultural land surface of the community (ha)

Average agricultural land plot owned by a household in the community (ha)

Largest agricultural land plot owned by a household in the community (ha)

Number of cattle heads

Number of cows

Number of sheep and goats

Average annual milk yield per cow (l) 
ments of animals across nearby community boundaries. Density of communities was derived with ArcGIS 9.1 (ESRI; Redlands, CA, USA) in-built kernel density model using $1 \mathrm{~km}^{2}$ cells. The bandwidth parameter for the kernel functions used to control the degree of smoothing of the estimated intensity surface was computed using the Sheather-Jones calculation method over all communities (Sheather and Jones, 1991). Bandwidth calculation was carried out using the SM (Bowman and Azzalini, 2007) package in the statistical software $\mathrm{R}$, version 2.9.1 (R Development Core Team, 2009).

\section{Statistical analyses}

A four-stage approach was adopted to describe and analyse the spatial distribution of communities that reported at least one brucellosis seropositive animal during the study period.

Firstly, the broad-scale characteristics of the spatial distribution of community-level brucellosis reports in cattle and small ruminants were visualised using the kernel intensity ratio method (Bithell, 1990). Two surfaces, representing the intensity of events, were constructed using a kernel smoothing function; the first (numerator) surface was based on all communities where at least one Brucella seropositive animal was reported, and the second (denominator) was based on all communities. The ratio of the intensity surface of positive communities to the intensity surface of all communities involved in this study provided a relief map showing the spatial variation in the proportion of communities with serological evidence of brucellosis. To account for the lack of information from neighbouring countries, we used a kernel smoothing algorithm that included a correction term for edge effects in the package SPATSTAT (Baddeley and Turner, 2005) in the statistical software R, version 2.9.1.

In parallel, areas of significantly greater risk of reporting serological evidence of brucellosis were identified by quantifying the amount of spatial correlation between communities' brucellosis status using the spatial scan statistic (Kulldorff and
Nagarwalla, 1995). This method is based on creating a series of circular windows of variable radii around every community with seropositives. Each circular window was set to contain a pre-determined fraction of the total set of communities involved in the study which was arbitrarily limited to $15 \%$. The proportion of communities with seropositives within each window was compared to the proportion of those outside the window. The scan statistic tested the significance of the frequency of disease within each window by assuming that the expected number of communities with records of brucellosis followed a Bernoulli distribution. The resulting likelihood ratios were sorted in descending order and the window with the largest maximum likelihood value designated as the area that 'most likely' constituted a spatial cluster. P-values were computed by comparing each window's likelihood ratio statistic with the expected distribution generated from re-labelling of the data using a Monte Carlo procedure. Spatial scan statistic calculations were performed using SaTScan software programme, version 8.0.1 (Kulldorff and Information Management Services, 2009).

Secondly, separate logistic regression models for cattle and small ruminants were developed to evaluate associations between community-level descriptors and the reported presence of at least one Brucella seropositive animal. For these analyses, the outcome $Y_{i j}$ was the report of at least one seropositive animal from the $i^{\text {th }}$ community during the $j^{\text {th }}$ year. Bivariate logistic regression models were used to assess the effects of putative community-level factors on serological evidence of brucellosis in communities. Factors associated with the outcome at an alpha level $<0.2$ were then included in a multivariate logistic regression model. Using a two-way, stepwise elimination process, we retained all uncorrelated factors, along with those with biologically plausible two-way interactions, in the multivariate model if they significantly improved model fit at an alpha level $<0.05$ using the likelihood ratio test (LRT). The Akaike information criterion (AIC) (Akaike, 1973) was used to determine which com- 
bination of variables best explained the data with the minimal number of covariates. Influential observations, identified by a Cook's distance value $\geq 0.5$ were removed from the data set to provide a more robust model (Armitage et al., 2001).

The fixed-effects models were extended to include random effect terms to account for correlation arising from repetitively testing the same community. Regression analyses were performed using a generalised linear model with multivariate normal random effect terms using Laplace approximation method (Breslow and Clayton, 1993; Pinheiro and Bates, 2000; Venables and Ripley, 2002) implemented in the LME4 package (Bates, 2007) in R, version 2.9.1. Predictive performances of the final models were evaluated with the receiver operating characteristic (ROC) curves.

Thirdly, binned semivariograms were constructed using the community-level residuals (Chambers and Hastie, 1992) to identify the presence of residual spatial autocorrelation in the data. Acknowledging that spatial autocorrelation may differ according to direction (Bailey and Gatrell, 1995), the presence of anisotropy (i.e. directional dependence) was evaluated by constructing semivariograms at angle classes of $0 \pm 22.5^{\circ}, 45 \pm 22.5^{\circ}, 90 \pm 22.5^{\circ}$, and $135 \pm$ $22.5^{\circ}$ (Isaacs and Srivistava, 1989). A series of 999 Monte Carlo simulations were carried out where the community-level residuals were randomly allocated to each community location and a semivariogram computed on each occasion. Pointwise minimum and maximum values of the simulated semivariograms were plotted as simulation envelopes.

Finally, maps of predicted probabilities and community-level residuals were plotted for the whole of the Republic of Armenia using the weighted kernel intensity ratio method (Baddeley and Turner, 2005). To compute these smoothed maps, two surfaces representing the intensity of events within the study area were constructed; the first (numerator) was the weighted intensity surface based on all communities where the weights were either the predicted probabilities of communities having seropositive animal or the community-level residuals, and the second (denominator) was the intensity surface based on all communities as previously computed. The ratio of these two intensity surfaces provided a relief map showing the spatial variation of either the predicted probabilities of serological evidence of brucellosis in communities or the extent to which each predicted estimates deviate from the observed values.

\section{Results}

\section{Descriptive analyses}

Of the 858 communities involved in this study, 326 communities (38\%; 95\% confidence interval (CI): $35 \%-41 \%$ ) reported serological evidence of brucellosis in at least one animal in 2006-2007. Two hundred and forty nine communities (29\%; $95 \%$ CI: $26 \%-32 \%$ ) reported seropositive cattle and 168 (21\%; 95\% CI: 18\%-24\%) reported seropositive small ruminants. Of those, $66(27 \%$; 95\% CI: $21 \%-32 \%)$ and 31 (19\%; 95\% CI: $13 \%-25 \%)$ communities reported serological evidence of brucellosis in both years in their cattle and small ruminant population, respectively. Of the 816 communities showing together records of cattle and small ruminant populations, $28(3.4 \%$; $95 \%$ CI: $2.4 \%-$ $4.9 \%)$ and 53 (6.5\%; $95 \%$ CI: $5.0 \%-8.4 \%)$ showed serological evidence of brucellosis in both large and small ruminants.

Community level prevalence surfaces of spatial patterns of brucellosis for cattle and small ruminants are shown in Figures 1a-1d. Computed smoothing parameters (i.e. the spatial extent of which each community influences its neighbourhood) were estimated to 10.0 and $10.4 \mathrm{~km}$ for cattle and small ruminants, respectively, almost thrice greater than the median nearest neighbour distance (3.20; IQR = 2.19-3.45). Three trends were evident in these two populations: (i) most of the communities reporting brucellosis in cattle and small ruminant were adjacent to the western border; (ii) serological evidence in communities remained relatively constant for cattle but increased over time for small ruminants; and (iii) in the south of the country, pro- 
(a)

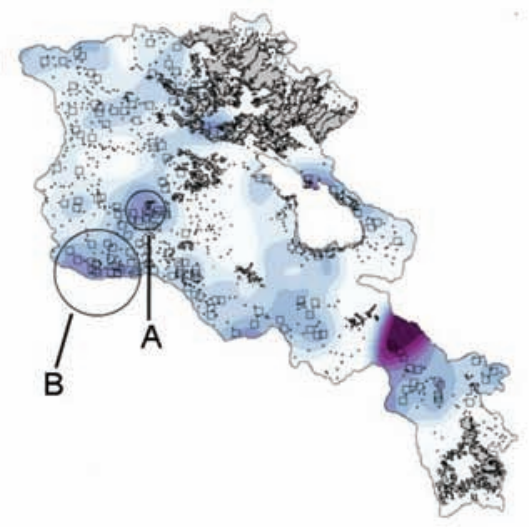

(c)

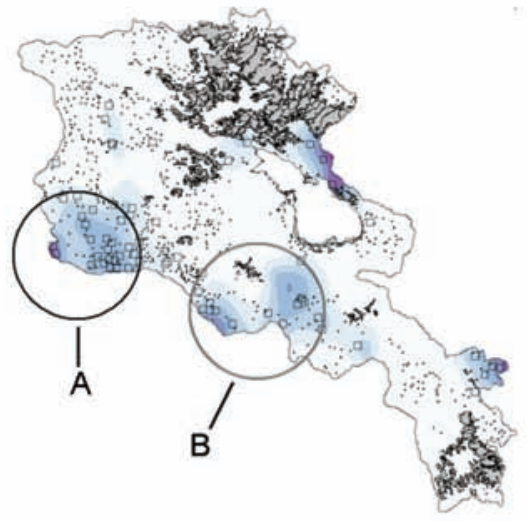

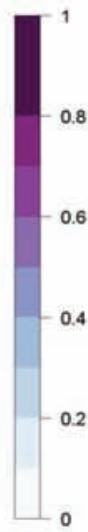

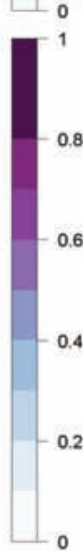

(b)

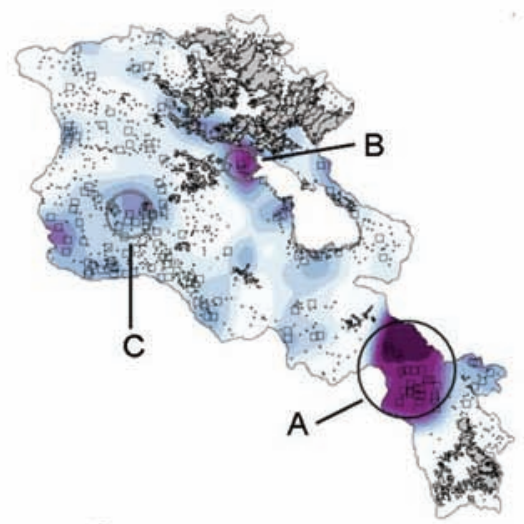

(d)

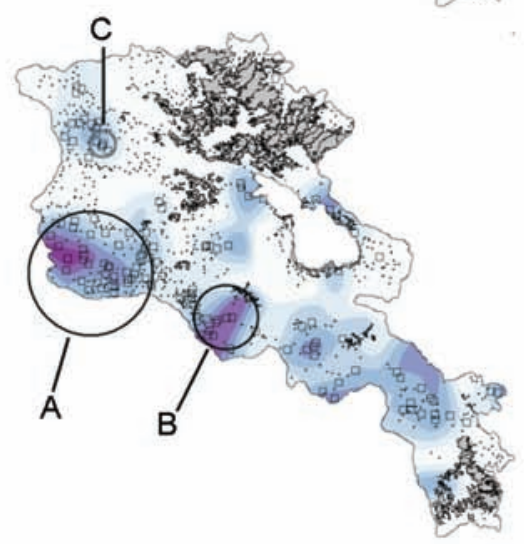

Fig. 1. Brucellosis in the Republic of Armenia, 2006-2007. Smoothed maps showing the smoothed spatial distribution of brucellosis community-level prevalence for communities where cattle (a, b) and small ruminants (c, d) were found infected in 2006 $(\mathrm{a}, \mathrm{c})$ and in 2007 (b, d). The large white polygon in the east of the country represents Lake Sevan. Dark polygons represent forested areas.

portions of communities reporting one or more seropositives were higher for cattle than for small ruminants.

Circles in Figure 1 show the location of areas that most likely constitute spatial clusters of communities with at least one brucellosis-seropositive animal in the Republic of Armenia for each year and for both animal populations. Numbers of communities in each cluster, the number of observed communities reporting brucellosis, and the odds ratio (OR) for positivity are all shown in Table 2. Areas detected as having significant greater risk of serological evidence of brucellosis in communities mostly support the observed pattern shown in Figure 1.

Analyses of scan statistics detected two areas of significantly greater odds for communities having at least one seropositive cattle in both 2006 and 2007. Although the locations of the northern "high-risk" area remained at least mildly significant $(\mathrm{P}<0.1)$ throughout the study period, the area in the south of the country was only at a significant greater odds in 2007 (Fig. 1, Table 2). The analyses detected one and two areas of significantly $(\mathrm{P}<0.05)$ greater odds for communities having at least one seropositive small ruminant in 2006 and 2007 respectively. Unlike the spatial pattern observed for cattle, all "high-risk" areas (OR >2) for communities having at least one seropositive small ruminants were located in the north-west of the country and coincide with areas of greater proportions of communities with brucellosis computed from kernel intensity ratio (Fig. 1). 
Table 2. Table 2. Brucellosis in the Republic of Armenia, 2006 - 2007. Details of the detected spatial high-risk areas for communities with evidence of brucellosis in their cattle and small ruminant populations during 2006 and 2007. Detected high-risk areas show potential for being spatial clusters of communities with at least one report of brucellosis.

\begin{tabular}{|c|c|c|c|c|c|c|c|}
\hline Risk areas & $\mathrm{N}$ & $n$ & Observed cases & OR & Radius $(\mathrm{km})$ & LRT & $\mathrm{P}$ \\
\hline \multicolumn{8}{|l|}{ Cattle } \\
\hline \multicolumn{8}{|l|}{2006} \\
\hline First most likely & 156 & 19 & 13 & $4.01^{\mathrm{a}}$ & 14.4 & 11.9 & 0.006 \\
\hline Second most likely & 156 & 46 & 21 & 2.75 & 30.8 & 9.8 & 0.040 \\
\hline \multicolumn{8}{|l|}{2007} \\
\hline First most likely & 159 & 32 & 25 & 4.82 & 34.8 & 28.3 & 0.001 \\
\hline Second most likely & 159 & 8 & 7 & 4.89 & 11.6 & 9.1 & 0.066 \\
\hline Third most likely & 159 & 25 & 14 & 3.22 & 16.5 & 9.1 & 0.068 \\
\hline \multicolumn{8}{|l|}{ Small ruminants } \\
\hline \multicolumn{8}{|l|}{2006} \\
\hline First most likely & 69 & 73 & 26 & 6.15 & 45.6 & 24.7 & 0.001 \\
\hline Second most likely & 69 & 55 & 15 & 3.84 & 48.4 & 9.3 & 0.067 \\
\hline \multicolumn{8}{|l|}{2007} \\
\hline First most likely & 130 & 110 & 43 & 3.17 & 44.4 & 20.7 & 0.001 \\
\hline Second most likely & 130 & 24 & 14 & 3.98 & 23.1 & 11.7 & 0.008 \\
\hline Third most likely & 130 & 5 & 5 & 6.49 & 9.7 & 9.3 & 0.085 \\
\hline
\end{tabular}

$\mathrm{N}=$ number of brucellosis-infected communities; $n$ = number of communities involved in the detected high-risk areas; Observed cases $=$ observed number of brucellosis-infected communities involved in the detected high-risk area; OR = odds ratio; LRT = likelihood ratio test. ${ }^{a}$ The interpretation is that communities involved in the detected high risk area were 4 times more likely to report at least one brucellosis-seropositive bovine than communities outside this area.

\section{Associated factors}

The five factors found to be associated with communities having at least one brucellosis-seropositive bovine are presented in Table 3. For each unit increase of $\log _{10}$ community cattle population size, the odds of a positive diagnosis increased by a factor of 4.07 (95\% CI: 2.13-7.81). Unit increases of $\log _{10}$ agricultural land size increased the odds of reporting at least one positive diagnosis by a factor 3.45 (95\% CI: $1.58-7.54$ ). Communities with $>10 \%$ of ethnic Yezidi (Armenia's largest minority who are mostly transhumant pastoralists depending on livestock and animal products for their livelihood) in their population were 3.05 times more likely $(95 \%$ CI: 1.77-5.26) to have evidence of infection than communities with a smaller proportion of Yezidi. The interpretation of the estimate of the OR for the "log-transformed community density" variable is that for every unit increase in $\log _{10}$ community density, which was set at 1 community per $100 \mathrm{~km}^{2}$, the odds of a community having evidence of infection increased by 4.49 (95\% CI: 1.51-13.4; Table 3). Communities with evidence of disease in small ruminants during the year of interest were almost 9 times more likely $(\mathrm{OR}=8.56$; 95\% CI: 3.22-22.8) to report at least one seropositive bovine than communities with no evidence of seropositive small ruminants. This latter effect was however conditional on the local density of communities $(\mathrm{P}=0.03)$ and that interaction effect can be explained as for every unit increase of $\log _{10}$ community density, the odds due to the presence of disease in sheep and goats decreased exponentially from the base effect of 8.56 .

Table 4 reports the eight variables associated with community level status for small ruminants. As with cattle, agricultural land size, more than $10 \%$ of eth- 
Table 3. Brucellosis in the Republic of Armenia, 2006-2007. Point estimates, standard errors (SE) of their regression coefficients, p-values $(\mathrm{P})$ and odds ratios (OR) and their $95 \%$ confidence intervals (CI) in a mixed-effects logistic regression model of factors associated with communities having at least one brucellosis-seropositive bovine.

\begin{tabular}{|c|c|c|c|c|}
\hline Variables & Estimate & SE & $\mathrm{P}$ & OR $(95 \% \mathrm{CI})$ \\
\hline Intercept & -10.70 & 1.34 & $<0.001$ & \\
\hline Log-transform community density (per $100 \mathrm{~km}^{2}$ ) & 1.50 & 0.56 & 0.007 & $4.49(1.51-13.4)$ \\
\hline \multicolumn{5}{|l|}{ Record of at least one seropositive sheep or goat } \\
\hline No & Ref. & & & 1.0 \\
\hline Yes & 2.15 & 0.50 & $<0.001$ & $8.56(3.22-22.8)$ \\
\hline Log-transform cattle population size & 1.41 & 0.33 & $<0.001$ & $4.07(2.13-7.81)$ \\
\hline Log-transform agricultural land surface area (ha) & 1.24 & 0.40 & 0.002 & $3.23(1.58-7.54)$ \\
\hline \multicolumn{5}{|l|}{ Proportion of Yezidi $\geq 10 \%$} \\
\hline No & Ref. & & & 1.0 \\
\hline Yes & 1.11 & 0.28 & $<0.001$ & $3.05(1.77-5.26)$ \\
\hline \multicolumn{5}{|l|}{ Interaction } \\
\hline \multicolumn{5}{|l|}{ Brucellosis in sheep or goats $x \log$ community density } \\
\hline No & Ref. & & & \\
\hline Yes & -1.81 & 0.85 & 0.032 & \\
\hline
\end{tabular}

$\mathrm{R}^{2}=0.08$. Variance (SD) of the random effect "Community" was $1.83(1.35)$.

Table 4. Brucellosis in the Republic of Armenia, 2006-2007. Point estimates, standard errors (SE) of the regression coefficients, p-values $(\mathrm{P})$ and odds ratios (OR) and their $95 \%$ confidence intervals (CI) in a mixed-effects logistic regression model of factors associated with communities having at least one brucellosis-seropositive small ruminant.

\begin{tabular}{|c|c|c|c|c|}
\hline Variables & Estimate & SE & $\mathrm{P}$ & OR $(95 \% \mathrm{CI})$ \\
\hline Intercept & -14.18 & 3.17 & $<0.001$ & \\
\hline \multicolumn{5}{|l|}{ Year } \\
\hline 2006 & Ref. & & & 1.0 \\
\hline 2007 & 0.98 & 0.120 & $<0.001$ & $2.67(1.80-3.93)$ \\
\hline \multicolumn{5}{|l|}{ Agricultural land $\geq 1217$ ha } \\
\hline No & Ref. & & & 1.0 \\
\hline Yes & 0.54 & 0.28 & 0.058 & $1.71(0.98-2.99)$ \\
\hline \multicolumn{5}{|l|}{ Proportion of Yezidi $\geq 10 \%$} \\
\hline No & Ref. & & & 1.0 \\
\hline Yes & 0.92 & 0.32 & 0.004 & $2.51(1.34-4.69)$ \\
\hline $\log _{10}$-transform number of inhabitants & 2.51 & 0.96 & 0.009 & $12.3(1.86-80.8)$ \\
\hline Elevation (x $100 \mathrm{~m})$ & 0.39 & 0.19 & 0.039 & $1.48(1.02-2.14)$ \\
\hline \multicolumn{5}{|l|}{ Record of at least one seropositive bovine } \\
\hline No & Ref. & & & 1.0 \\
\hline Yes & 2.16 & 0.53 & $<0.001$ & $8.66(3.08-24.4)$ \\
\hline $\log _{10}$-transform sheep and goat population size & 1.93 & 0.29 & $<0.001$ & $6.29(3.90-12.2)$ \\
\hline $\log _{10}$-transform distance from the Marz centre $(\mathrm{km})$ & -0.73 & 0.34 & 0.031 & $0.48(0.25-0.93)$ \\
\hline $\log _{10}$-transform community density (per $100 \mathrm{~km}^{2}$ ) & -0.02 & 0.56 & 0.973 & $0.98(0.33-2.92)$ \\
\hline \multicolumn{5}{|l|}{ Interactions: } \\
\hline Log number of inhabitants $x$ elevation & -0.16 & 0.060 & 0.007 & \\
\hline \multicolumn{5}{|l|}{ Brucellosis in cattle $\times \log$ community density } \\
\hline No & Ref. & & & \\
\hline Yes & -1.95 & 0.90 & 0.030 & \\
\hline
\end{tabular}

$\mathrm{R}^{2}=0.17$. Variance (SD) of the random effect “Community” was 1.45 (1.21). 
nic Yezidi in a community, diagnosis of brucellosis in cattle, and $\log _{10}$-transformed small ruminant population size were significantly associated with the community-level odds of reporting at least one seropositive small ruminant. Additional factors were the year of surveillance, elevation above sea level, the $\log _{10}$-transformed distance of the community from the Marz (i.e. district) administrative centre and the $\log _{10}$-transformed number of inhabitants in the community. Each unit increase of $\log _{10}$ number of inhabitants in a community increased the odds of having at least one seropositive small ruminant by a factor of 8.66 (95\% CI: 3.08-24.4) but this association was conditional on the altitude of the community. As elevation above sea level increased, the influence of the number of inhabitants in communities significantly decreased for every unit increase in density of communities (results not shown).

Empirical semivariograms computed using the community-level residuals lay entirely within the Monte-Carlo simulation envelopes, indicating an absence of unexplained spatial dependency in the data (Fig. 2). Identifying the same correlation patterns over increasing distances for different directions showed that the observed lack of spatial dependence

(a)

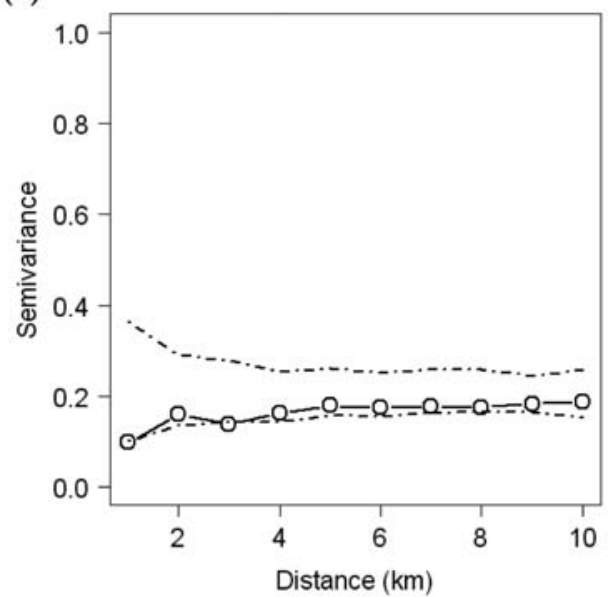

in the residuals did not vary with direction.

Random effects computed in both models showed significant correlation patterns at the community level, indicating a strong dependency in the data due to repeated testing of the same communities.

\section{Predicting abilities and accuracy}

Although a small proportion of the observed variability was explained by both models $\left(R^{2}<0.2\right)$, the area under the ROC curve (AUC) was substantial (AUC >0.9; Fig. 3). This result indicates that both models were able to correctly discriminate between the observed presence and absence of at least one Brucella-seropositive animal in Armenian communities $94.4 \%$ and $92.7 \%$ of the time for cattle and small ruminants, respectively. Specificity and sensitivity were optimal for diagnosing a community as having at least one record of a seropositive animal for predicted probabilities greater than 0.153 and 0.130 for cattle and small ruminants, respectively. Sensitivity, specificity and predictive values of the predictive models (Table 5) using such cut-off points for declaring a given community as having at least one test-positive in their cattle and small ruminant population are shown in Figure 3.

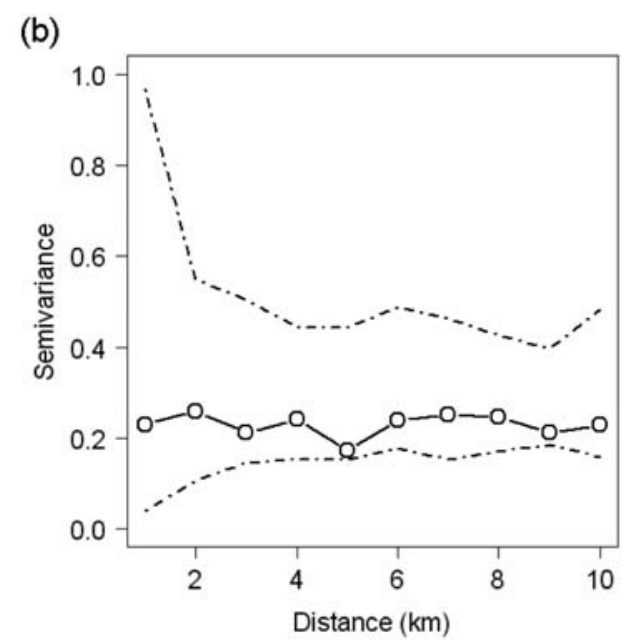

Fig. 2. Brucellosis in the Republic of Armenia, 2006-2007. Binned, empirical semivariograms computed using the standardised residuals derived from the mixed-effects logistic regression model of factors associated with risk for communities having at least one seropositive (a) bovine, and (b) small ruminant. 
(a)

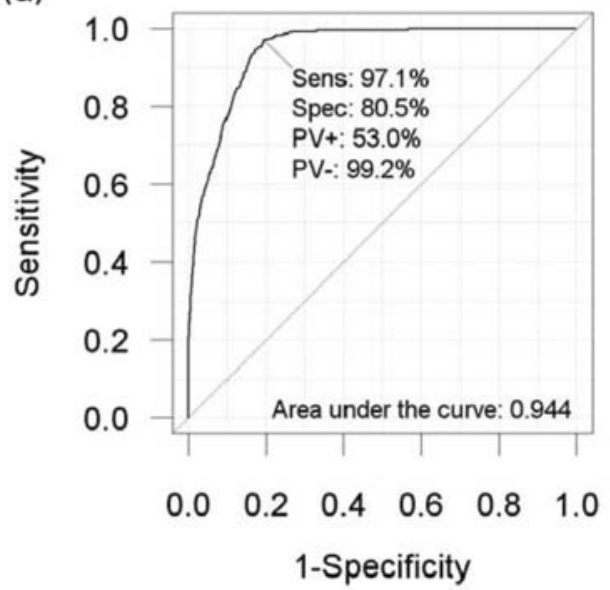

(b)

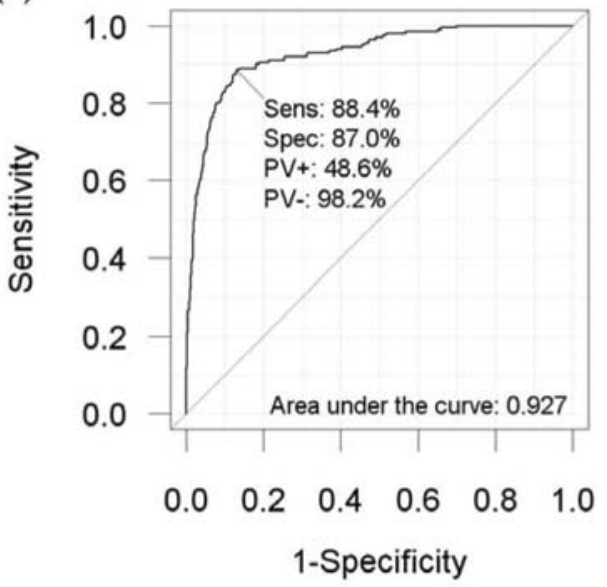

Fig. 3. Brucellosis in the Republic of Armenia, 2006-2007. Line plots showing the receiver operating characteristic (ROC) curve testing the predictive ability of the mixed effects logistic regression model of factors influencing the community-level risk of brucellosis occurrence in (a) cattle and (b) small ruminants. The area under the ROC curve (AUC) is often used as a single threshold-independent measure for model performance. The AUC ranges from 0.5 to 1 , where 1 indicates perfect prediction and values of 0.5 indicate poor predictive ability. Values can be classified as: poor $(0.5 \leq \mathrm{AUC}<0.7)$, good $(0.70 \leq \mathrm{AUC}<0.9)$ and excellent predictive ability (AUC $\geq 0.9$ ). Sensitivity (sens), specificity (spec) and predictive values (positives ( $\mathrm{pv}+$ ) and negatives (pv-)) of the optimal cut-points for declaring a community brucellosis-infected are also indicated.

Table 5. The confusion matrix used to evaluate the accuracy of the two mixed-effects logistic regression models of factors associated with communities reporting one or more brucellosis-seropositive animals. TP $=$ number of communities for which presence of brucellosis report was correctly predicted by the model; FP = number of communities for which there was no evidence of brucellosis but the model predicted its occurrence; $\mathrm{FN}=$ number of communities for which evidence of brucellosis was found but the model predicted absence of disease; $\mathrm{TN}$ = number of communities for which absence of evidence of brucellosis was correctly predicted by the model.

\begin{tabular}{|c|c|c|c|c|}
\hline & & \multicolumn{2}{|c|}{$\begin{array}{l}\text { Communities reporting serological } \\
\text { evidences of brucellosis }\end{array}$} & \\
\hline & & Yes & No & \\
\hline \multirow{3}{*}{$\begin{array}{l}\text { Model } \\
\text { outcome }\end{array}$} & $p \geq k$ & $\mathrm{TP}$ & FP & $\mathrm{PV}+=\mathrm{TP} /(\mathrm{TP}+\mathrm{FP})$ \\
\hline & $p<k$ & $\mathrm{FN}$ & $\mathrm{TN}$ & $\mathrm{PV}-=\mathrm{TN} /(\mathrm{FN}+\mathrm{TN})$ \\
\hline & & $\begin{array}{c}\text { Sensitivity } \\
=\mathrm{TP} /(\mathrm{TP}+\mathrm{FN})\end{array}$ & $\begin{array}{c}\text { Specificity } \\
=\mathrm{TN} /(\mathrm{FP}+\mathrm{TN})\end{array}$ & \\
\hline
\end{tabular}

$p=$ predicted probability for communities reporting brucellosis; $k=$ optimal cut-off probability; PV+ $=$ positive predictive values; PV- = negative predictive values.

\section{Localisation of at-risk areas}

The predicted probabilities of communities having at least one Brucella-seropositive animal in their cattle populations were $32.5 \%$ (95\% CI: $29.5-35.7 \%$ ) and $35.2 \%$ (95\% CI: 32.1-38.5\%) in 2006 and in
2007, respectively. The corresponding respective probabilities for at least one seropositive small ruminant were $14.0 \%$ (95\% CI: $11.8-6.5 \%)$ and $30.3 \%$ (95\% CI: $27.2-33.5 \%$ ).

Figures 4 and 5 depict the smoothed fitted community-level probabilities of serological evidence of 


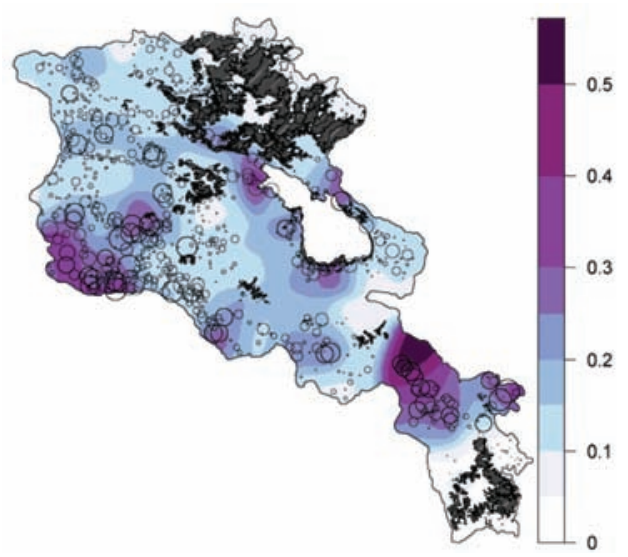

Fig. 4. Brucellosis in the Republic of Armenia, 2006-2007. Smoothed maps showing the smoothed spatial distribution of the predicted probability of occurrence computed from the mixed-effects logistic regression model of factors associated with the community-level risk of brucellosis occurrence in cattle. The size of the circles represents the community-level predicted probability of brucellosis occurrence. The large white polygon in the east represents Lake Sevan. Dark polygons represent forested areas.

(a)

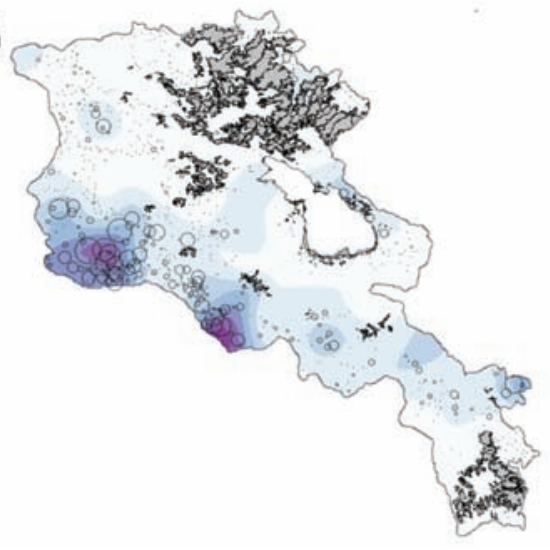

brucellosis throughout the Republic of Armenia for cattle and small ruminants. Areas predicted as having greater risk of brucellosis support the observed pattern shown in Figure 1. Communities in two areas of the country were predicted to be at greater risk for their cattle populations to test positively, one in the south of the country, and the other along the north-western border. Corresponding areas of greater risk for small ruminant populations were predicted in two areas along the western border.

Smoothed maps of model residuals for cattle and small ruminants are shown in Figure 6. The maps illustrate the ranges in positive and negative directions from large to null in the amount of deviance of the predicted average trend from the observed community status. Four areas where the community-level risk was poorly predicted can be seen in Figure 6 . They are illustrated with solid contours (contour set to surround areas with residual $\geq 0.4$ ): one large area for cattle in the south, and three others for small ruminants. The first is located between the northeastern border and Lake Sevan, the second is located in the middle of the country, while the third is a small area in the south.

Fig. 5. Brucellosis in the Republic of Armenia, 2006-2007. Smoothed maps showing the smoothed spatial distribution of the predicted probability of occurrence computed from the mixed-effects logistic regression model of factors associated with the community-level risk of brucellosis occurrence in either sheep or goats in: (a) 2006, and (b) 2007. The size of the circles represents the community-level predicted probability of brucellosis occurrence. The large white polygon in the east represents Lake Sevan. Dark polygons represent forested areas. 
(a)

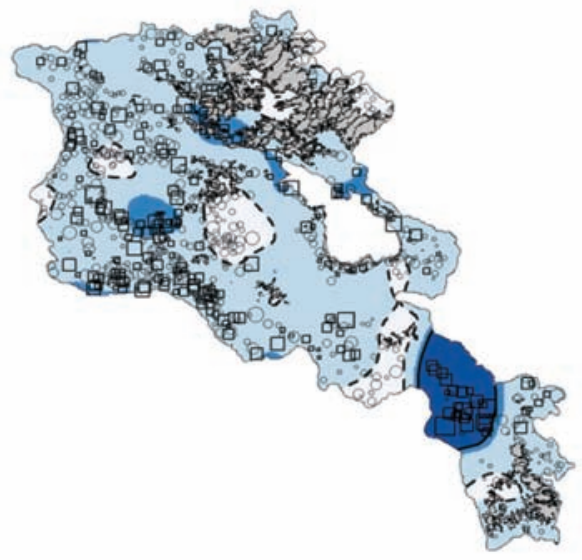

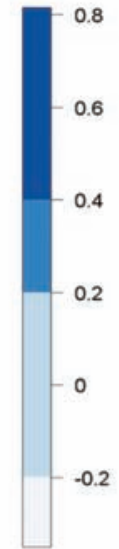

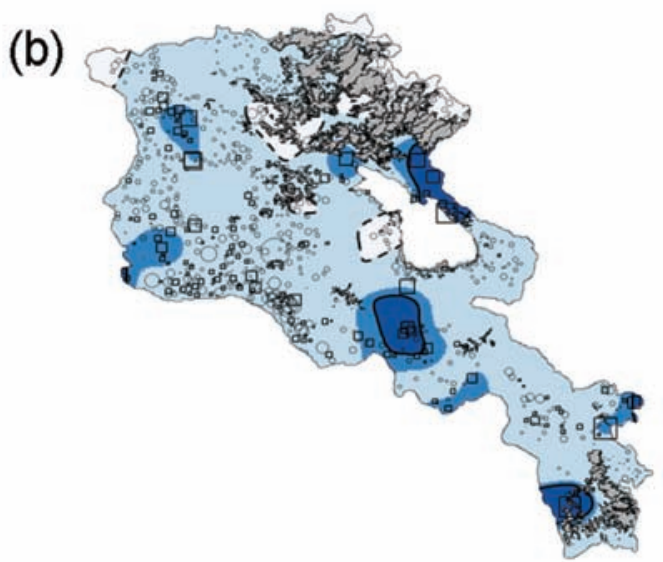

Fig. 6. Brucellosis in the Republic of Armenia, 2006-2007. Smoothed maps showing the smoothed spatial distribution of the community-level residuals from the mixed effects logistic regression model of factors associated with the community-level risk of brucellosis occurrence in (a) cattle, and (b) small ruminants. Graduated colours indicate the areas where the model either over-predicts (light colour) or under predicts (dark colour). Square and circle symbols represent communities where the model either under- or over-predicts, respectively. The size of the symbols represents the value of the community-level residual. The large white polygon in the east of the country represents Lake Sevan. Dark polygons represent forested areas.

\section{Discussion}

This study aimed to improve the understanding of brucellosis epidemiology in the Republic of Armenia. The analysis of data from the current national brucellosis control programme was undertaken with that objective firmly in mind and an expectation for more robust findings for some features of the disease than those that could be obtained from the recent FAO-Government of Armenia (GOA) national seroprevalence survey (Jackson and Porphyre, 2008). The data used in this study were considered a valuable resource, though imperfect, for gaining insights into the nature of the disease at community level given the large number of infected communities with information about species involved and date of the recorded detection. The analyses essentially sought to identify high-risk locations and factors that might be associated with community-level risky practices in order that they might be given special consideration for control and surveillance operations.

The data used in these analyses were produced under less than ideal conditions. The collapse of the
Soviet Union in 1991 brought independence to the Republic of Armenia but also introduced major economic and socio-political problems which seriously affected the delivery of public services (von SchoenAngerer, 2004). The RBT is considered to be a robust test but it is likely that its normally high sensitivity (MacMillan, 1997; World Organisation for Animal Health, 2008) would have been adversely affected, albeit probably mildly, by a lack of standardised reagents and poorly maintained laboratory facilities and equipment during 2006 and 2007. Furthermore, the testing component of the national brucellosis control programme focused mainly on positive reactions and denominator test data were not always recorded for communities in the central database when there were no positive test results. This procedure has now been corrected, but it presented some difficulties for the analysis. To overcome these constraints to some extent, we limited the outcome of interest to communities, and evidence of infection to reports of one or more seropositives in their domestic animal populations. This approach was based on assurances from personal examination of records in several regional lab- 
oratories by three of the authors and discussions with field veterinarians and livestock owners as well as senior administration veterinarians, that most of the communities involved in the brucellosis testand-slaughter programme were visited at least once each year during the study period. However, it was also apparent that all animals in the communities were sometimes not always tested for various reasons, including absence during migrations, animals not always held back from outside grazing for testing and inadequate resources. Misclassification of community status due to false positives, even when only one positive was recorded, was unlikely because the complement fixation test is generally regarded as very close to $100 \%$ specific. The data were used for training of staff of the epidemiology unit in the FS\&SVI and as such were subject to close scrutiny and cleared of data entry errors and inconsistent spelling of community names. In addition to limiting analytical constraints, communities were considered important because of the considerable mixing of animals and opportunities for transmission of infection through communal grazing, trade and loan of animals.

Despite the uncertainty regarding biases in the data, the observed proportion of communities with evidence of brucellosis in their livestock was in close agreement with results from the FAO-GOA crosssectional serological survey conducted in February and March 2008 (Jackson and Porphyre, 2008). That survey involved 80 randomly selected communities of which $36.3 \%$ (95\% CI: 26.6-47.2\%) showed evidence of infection detected by primary RBT and confirmatory testing of positives with a competitive, enzyme-linked immunosorbent assay (cELISA). In that study, collection of samples was closely supervised and all tests were done at a central laboratory under strictly controlled conditions. The analyses essentially sought to identify high-risk locations and factors that might be associated with community-level risky practices.

Density of communities, communities' animal population size and agricultural land size, proportion of ethnic Yezidi, and presence of infection in other species were associated with the probability of communities having seropositive cattle or small ruminants. This association with ethnicity is interesting and more investigation on this finding may identify particular husbandry practices that put those flocks and herds at greater risk. The overall volume of their sales of sheep, goat and cattle cheese and dairy products is unknown but may be disproportionately higher than that produced by Armenian predominant communities.

The study showed evidence of similarities, but also some differences, in the epidemiological patterns of the disease in large and small ruminants (Tables 3 and 4), one of them being the relative importance of elevation above sea level of the community. Elevation was found to be significantly associated with the presence of at least one seropositive small ruminant in communities. For each $100 \mathrm{~m}$ increases in elevation above sea level, the odds of communities with serological evidence of brucellosis in small ruminants increase by a factor $1.48(95 \%$ CI: 1.02-2.14). This finding may be a proxy effect due to longer periods of confinement in barns during winter periods at higher altitudes leading to more opportunity for disease transmission under conditions of close confinement and contact.

Although the spatial pattern of community-level occurrence of brucellosis in cattle was constant throughout the study period, it contrasted with the pattern observed for small ruminants (Figs. 1, 4 and 5 ), with a greater predicted probability of Brucella serological evidence in communities' small ruminants in 2007 (30\%) than in 2006 (14\%; Table 4). Two non-exclusive explanations may be suggested: (i) the epidemiology of the disease in cattle and small ruminants is likely to be very different because of the influences of separate species of Brucella, not only B. melitensis and B. abortus but also B. ovis, and dissimilar routes of infection, and (ii) the intensity of the surveillance programmes for the two populations may have differed due to economic or logistic constraints. Patterns of disease in cattle and small ruminants observed in the FAO-GOA serosurvey (Jackson and Porphyre, 2008) tend to suggest 
that in some areas there were mixed infections of $B$. abortus and B. melitensis operating in cattle and small ruminants. However it also seemed that on some occasions single species of Brucella predominated. In the absence of sound information about the management of the testing programme, and lack of details of husbandry and management of both populations at individual flock and herd levels, the reasons for the different epidemiological patterns must remain speculative.

In our attempt to evaluate the predictive performance of the models, substantial AUC values of $>0.90$ were obtained, indicating that both models were able to correctly discriminate between the observed presence and absence of at least one Brucella seropositive animal in Armenian communities $94.4 \%$ and $92.7 \%$ of the time for cattle and small ruminants, respectively. However, several features call for caution in the interpretation of these findings. First, to declare from our analyses a community as potentially reporting a seropositive bovine or small ruminant, models should predict respective probabilities greater than the relatively low cut-off points of 0.15 and 0.13. Second, although these cut-off points optimised both sensitivity and specificity, weak positive predictive values were recorded (Fig. 3): almost half of the predicted communities that would show evidence of brucellosis in their livestock population were indeed recorded with seropositive animals. These outcomes, together with the fact that less than $20 \%$ of the variations in the data are explained in the model, further tends to indicate that other factors are involved in the reporting of evidence of brucellosis in communities. Another hypothesis is that some communities presented no serological evidence of brucellosis despite being infected due to a less than perfect screening of the animals' disease status within communities.

Several areas were identified as having a probability of occurrence of positive serological tests which was poorly explained by the influential risk factors. In Figure 6, two types of areas can be distinguished: areas where (i) communities with no observed evi- dence of brucellosis but with relatively large predicted probability of occurrence (represented with clear shade and dashed contour), and (ii) communities with observed evidence of brucellosis but with relatively small predicted probability of occurrence (represented with dark shade and solid contour). This discrepancy indicates a need for further studies to determine the reasons for predictions differing from observations. These findings, together with findings of strong dependency in the data due to repeated testing of the same communities (Tables 3 and 4), point in the direction of obtaining a better understanding of disease prevalence as well as factors that influence the occurrence of the disease at household level and which might be targeted for control programme interventions.

Problems of the models' false positive aside, the excellent $(>98 \%)$ negative predictive values using the determined cut-off points obtained from both models give some level of confidence for the areas predicted as showing a low level of disease circulation. This could allow delineation of some specific geographical zones where surveillance and control methods, such as test-and-slaughter and vaccination programmes which are more suited to low levels of disease might be implemented.

A long-term commitment to an integrated riskbased, national control programme will be required to reduce the prevalence of the disease in livestock and humans. Other investigations will be needed to quantify aspects of husbandry and animal health practices such as the extent of out-of-season breeding, animal housing conditions, and movements and trading of animals that represent high risk practices. It is important to recognise that the factors and 'high-risk' locations identified in these analyses are based on statistical measures of their association with serological evidence of disease and do not provide proof of causal associations. Notwithstanding this caution, factors that were biologically plausible and were identified in all analyses can be regarded as the best indicators of risk at present and as such warrant serious consideration for the control programme. The factors identified in this study make a 
good starting place for information-gathering at a finer level of detail.

\section{Conclusion}

In summary, despite not knowing at this stage which Brucella species were involved, it can be stated that the national control data showed a widespread and uneven distribution of serological evidence of brucellosis throughout Armenian communities. However, comparison of the findings for cattle and small ruminants showed enough differences to suggest that different epidemiological forces may be operating for those two populations. We also conclude that, despite less than perfect data, these analyses identified priority areas for the implementation of targeted risk-based surveillance and control measures.

\section{Acknowledgments}

The authors gratefully acknowledge funding from the Food and Agriculture Organization (FAO) and the Government of Italy under project "Phase I - Assistance to Brucellosis Control in Armenia" (GCP/ARM/001/ITA) and the use of resources of the EpiCentre, Institute of Veterinary, Animal and Biomedical Sciences, Massey University, Palmerston North, New Zealand. In particular we thank, Nedzad Ajanovic and Andiry Rozstalnyy, FAO-Budapest, Armen Sedrakyan, FAO-Yerevan, and Ahmed El-Idrissi, FAO-Rome.

\section{References}

Akaike H, 1973. Information theory as an extension of the maximum likelihood principle. Proceedings of the 2nd International Symposium on Information Theory, Budapest, Hungary, pp. 267-281.

Anonymous, 2008. Brucellosis - incidence [on line]. Centralized Information System for Infectious Diseases (CISID) of the World Health Organization (WHO) Regional Office for Europe. http://data.euro.who.int/cisid (accessed: 9 February 2009).

Armitage P, Berry G, Matthews J, 2001. Statistical methods in medical research. Blackwell Scientific Publication,
Oxford, UK, pp. 832.

Baddeley A, Turner R, 2005. Spatstat: an R package for analyzing spatial point patterns. J Stat Softw 12, 1-42.

Bailey T, Gatrell A, 1995. Interactive spatial data analysis. John Wiley \& Sons, New York, NY, USA, pp. 413.

Bates D, 2007. Lme4: Linear mixed-effects models using S4 classes. R package version 0.99875. http://lme4.r-forge.rproject.org/ (accessed: October 2009).

Bithell JF, 1990. An application of density estimation to geographical epidemiology. Stat Med 9, 691-701.

Bowman AW, Azzalini A, 2007. R package sm: nonparametric smoothing methods (version 2.2). University of Glasgow, UK and Universita di Padova, Italia. http://www.stats.gla.ac.uk/ adrian/sm and http://azzalini. stat.unipd.it/Book_sm (accessed: October 2009).

Breslow N, Clayton D, 1993. Approximate inference in generalized linear mixed models. J Am Stat Assoc 88, 9-25.

Chambers JM, Hastie T, 1992. Statistical Models in S. Wadsworth \& Brooks/Cole.

Hovhannisyan SG, 2004. Health care in Armenia - economic and sociopolitical problems mean the healthcare system is in transition. BMJ 329, 522-523.

International Centre for Tropical Agriculture, 2004. Voidfilled seamless SRTM data V1. Available from the CGIARCSI SRTM 90m Database. http://srtm.csi.cgiar.org and http://www.ambiotek.com/topoview (accessed: February 2008).

Isaacs E, Srivistava R, 1989. Introduction to applied geostatistics. Oxford University Press, London, UK, pp. 374.

Jackson R, Porphyre T, 2008. Republic of Armenia brucellosis seroprevalence report. Food and Agriculture Organization of the United Nations (FAO), 11- 42.

Kulldorff M, Nagarwalla N, 1995. Spatial disease clusters: detection and inference. Stat Med 14, 799-810.

Kulldorff M and Information Management Services, 2009. SaTScanTM v8.0: Software for the spatial and space-time scan statistics. http://www.satscan.org/ (accessed: October 2009).

MacMillan AP, 1997. Investigation of the performance of the Rose Bengal plate test in the diagnosis of Brucella melitensis infection in sheep and goats. World Animal Review 57-60.

Pappas G, Papadimitriou P, Akritidis N, Christou L, Tsianos EV, 2006. The new global map of human brucellosis. Lancet Infect Dis 6, 91-99. 
Pinheiro J, Bates D, 2000. Mixed-effects models in S and SPLUS. Springer-Verlag New York, NY, USA.

R Development Core Team, 2009. R: a language and environment for statistical computing. $\mathrm{R}$ Foundation for Statistical Computing, Vienna, Austria. http://www.R-project.org (accessed: October 2009).

Sheather S, Jones M, 1991. A reliable data-based bandwidth selection method for kernel density estimation. J R Stat Soc Series B Stat Methodol 53, 683-690.
Venables W, Ripley B, 2002. Modern applied statistics with S. Springer-Verlag, New York, NY, USA.

von Schoen-Angerer T, 2004. Understanding health care in the south Caucasus: examples from Armenia. BMJ 329, 562-565.

World Organisation for Animal Health, 2008. Manual of diagnostic tests and vaccines for terrestrial animals 2008. World Organisation for Animal Health (OIE), Paris, France, pp. 1343. 